\title{
Cost and effectiveness of omega- 3 fatty acid supplementation in Chinese ICU patients receiving parenteral nutrition
}

This article was published in the following Dove Press journal:

ClinicoEconomics and Outcomes Research

26 June 2015

Number of times this article has been viewed

\author{
Guo Hao Wu' \\ Jian $\mathrm{Gao}^{2}$ \\ Chun Yan Ji ${ }^{2}$ \\ Lorenzo Pradelli ${ }^{3}$ \\ Qiu Lei $\mathrm{Xi}^{\prime}$ \\ Qiu Lin Zhuang' \\ 'Department of General Surgery, \\ ${ }^{2}$ Department of Nutrition, Zhongshan \\ Hospital, Fudan University, Shanghai, \\ People's Republic of China; ${ }^{3}$ AdRes \\ Health Economics and Outcomes \\ Research, Piazza Carlo Emanuele II, \\ Torino, Italy
}

Background and objectives: Clinical evidence supports the use of omega-3 polyunsaturated fatty acid (PUFA)-enriched lipid emulsions in place of standard lipid emulsions in parenteral nutrition (PN) for intensive care unit (ICU) patients, but uptake may be limited by higher costs. We compared clinical and economic outcomes for these two types of lipid emulsion in the Chinese ICU setting.

Methods: We developed a pharmacoeconomic discrete event simulation model, based on efficacy data from an international meta-analysis and patient characteristics, resource consumption, and unit costs from a Chinese institutional setting. Probabilistic sensitivity analyses were undertaken to assess the effects of uncertainty around input parameters. Model predictive validity was assessed by comparing results with data observed in a patient subset not used in the modeling.

Results: The model predicted that omega-3 PUFA-enriched emulsion (Omegaven ${ }^{\circledR} 10 \%$ fish oil emulsion) would dominate standard lipid emulsions, with better clinical outcomes and lower overall health care costs (mean savings $\sim 10,000 \mathrm{RMB}$ ), mainly as a result of faster recovery and shorter hospital stay (by $\sim 6.5$ days). The external validation process confirmed the reliability of the model predictions.

Conclusion: Omega-3 PUFA-enriched lipid emulsions improved clinical outcome and decreased overall costs in Chinese ICU patients requiring PN.

Keywords: omega-3 PUFA-enriched lipids, ICU patients, total costs, microsimulation, external validation, length of hospital stay

\section{Introduction}

Malnutrition in hospital patients is a global concern: up to $50 \%$ of inpatients are malnourished or at risk for malnutrition. ${ }^{1-6}$ This is also true for the People's Republic of China, where figures of undernutrition in hospital patients are close to $10 \%{ }^{7}$ In fact, the overall percentage of hospitalized patients at nutritional risk, about $40 \%-50 \%$, is very similar to that observed in Western countries, as shown in a comparative survey of nutritional status conducted in 2006-2007 at a large hospital in the People's Republic of China and one in the USA. ${ }^{8}$ In the People's Republic of China and in other countries, malnourished patients fare worse than well-nourished individuals, recovering more slowly, with an increased risk of infection and mortality, and longer average length of hospital stay (LOS). ${ }^{9-14}$ Infections and longer LOS clearly result in excess resource consumption. Appropriate interventions aimed at preventing and treating malnutrition can potentially reduce the infection rate, complications, and hospital LOS and improve survival, thus conferring clinical and economic benefits. ${ }^{15,16}$

In critical illness, enteral nutrition is often not feasible, sufficient, or indicated, and thus parenteral nutrition $(\mathrm{PN})$ is required. Parenteral lipid emulsions are an integral
Correspondence: Guo Hao Wu ent of General Surgery, Zhongshan Hospital, Fudan University, People's Republic of China Email prowugh@।63.com

Jian Gao

Department of Nutrition, Zhongshan Hospital, Fudan University, I 38 Yixueyuan Road, Shanghai 200032, People's Republic of China

Email gao.jian@zs-hospital.sh.cn 
part of PN. Traditionally, they are prepared with vegetable oils rich in omega- 6 fatty acids, such as soybean oil, which tend to favor a proinflammatory state. More recently, the beneficial effects of $\mathrm{PN}$ enriched with omega-3 polyunsaturated fatty acids (PUFA), most commonly fish oils, have been demonstrated, especially in those conditions associated with inflammation, such as sepsis and pancreatitis. ${ }^{17}$ The potential of omega-3 PUFA to favorably modulate inflammation and hasten recovery and hospital discharge is acknowledged in the latest nutrition guidelines, which recommend their use in PN for both intensive care and surgical patients. ${ }^{18,19}$

The aim of this study was to compare clinical and economic outcomes for omega-3 PUFA-enriched versus standard lipid emulsions in the Chinese ICU setting, from the perspective of the patient and their family, reflecting the organization of the Chinese health care system. We used a discrete event simulation (DES) model based on efficacy data from an international meta-analysis and the People's Republic of China-specific clinical and economic input parameters derived from an observational study conducted in a large hospital based in Shanghai. ${ }^{17,20}$

The logical construct of the evaluation is based on estimating the effectiveness of the intervention by applying relative efficacy data from randomized controlled trials to patient outcomes as observed in real-world practice. The validity of this rationale will be tested by formal comparison of predicted data with data not used in the modeling exercise.

\section{Material and methods Model structure}

The model was based on the DES technique and developed in Excel. ${ }^{21}$ Two treatment arms were simulated and compared at patient-level: PN using standard lipid emulsions (ie, those not enriched with omega-3 PUFA; standard arm) versus PN supplemented by an omega-3 PUFA-enriched lipid emulsion (Omegaven ${ }^{\circledR} 10 \%$ fish oil [Fresenius Kabi, Bad Homburg, Germany]; Omegaven ${ }^{\circledR}$ arm). Each simulated ICU patient was concurrently sent to the Omegaven ${ }^{\circledR}$ and standard arms, ie, the simulation of the two alternatives ran on the same cohort. The simulation was run over 10,000 iterations, each ideally representing a patient. No discounting was applied to outcomes and costs due to the short time frame of the simulation, which covered only the duration of the hospital stay.

\section{Events and outcomes}

The following events were considered: transfers between ICU and ward, new nosocomial infection, discharge from the hospital, and death. The latter two events determined the end of the patient pathway. Simulated patients enter the model with ICU admission, directly or from the ward. Pre-ICU time did not differ between the two arms. In ICU, three alternative options were possible: the patient could be transferred to a general ward, discharged directly to home, or die in the ICU. For those in the ward there were two possibilities: either they could be discharged, or die (Figure 1).

\section{Clinical inputs}

The clinical input parameters for the control patient population were obtained by analyzing data collected at the Department of General Surgery, Zhongshan Hospital, Fudan University, Shanghai, People's Republic of China, from January 2008 to December 2011, as part of a clinical-economic trial that has been previously described and published. ${ }^{19}$ The full dataset comprised retrospective records from 1,101 patients from the general surgery service receiving PN supplemented with omega-3 PUFA-enriched lipid emulsions $\left(\right.$ Omegaven $^{\circledR}$ ) or

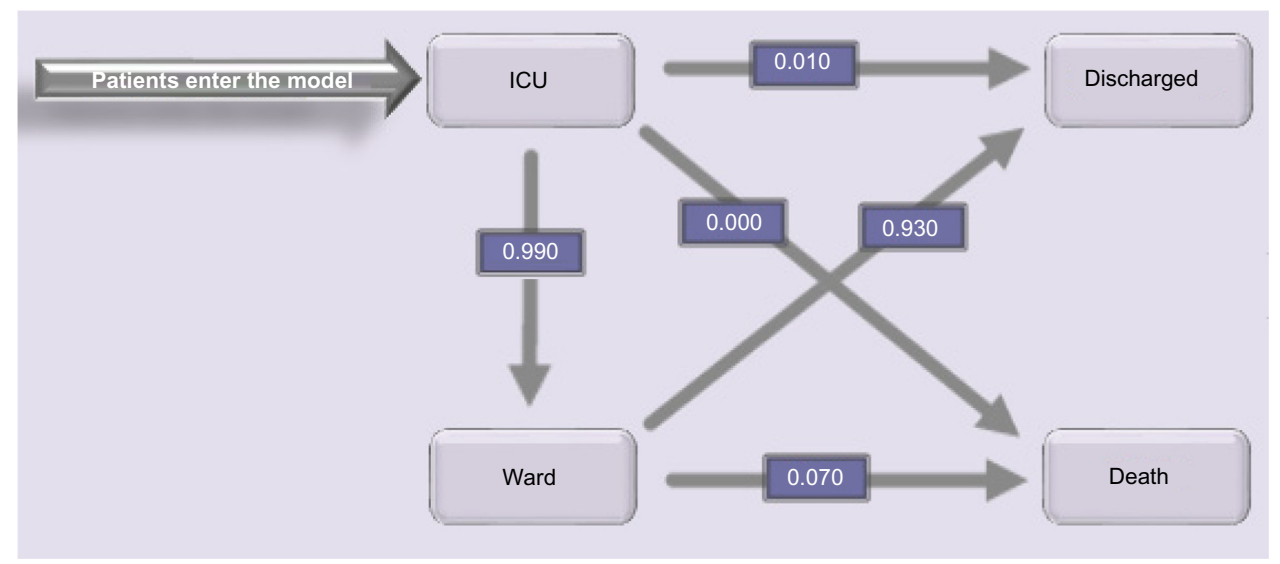

Figure I Model structure showing transitional probabilities, valid for the control population, derived from the hospital database. Abbreviation: ICU, intensive care unit. 
standard PN (with standard lipid emulsions) and matched 2:1 based on sex, age, main disease, ICU admission, and surgical procedure. The calorie requirements for ICU patients in our institution are routinely calculated according to the guidelines of the Chinese Society for Parenteral and Enteral Nutrition, ie, $20-25 \mathrm{kcal} / \mathrm{kg} / \mathrm{d}$ in acute stress period and $25-30 \mathrm{kcal} / \mathrm{kg} / \mathrm{d}$ thereafter. Nutritional emulsion doses were calculated after taking into account the caloric contribution of infused propofol and dextrose and, in the intervention group, of the supplemental n-3 emulsion, in order to assure correct and comparable energy delivery in both groups.

The subset of patients who were admitted to the ICU ( $n=443$ patients; 136 receiving Omegaven ${ }^{\circledR}$ and 307 not) were included for the current analysis. According to the clinical protocol, as reported in the publication, the primary statistical analyses were conducted on the population that received at least one PN infusion and the secondary analyses on the population of only patients who received at least 5 days of $\mathrm{PN}$, yielding comparable results. ${ }^{20}$

Clinical outcomes (mortality, infection rate, lengths of stay in ward and in ICU) of patients who were admitted to ICU and received standard $\mathrm{PN}$ were used for modeling the standard arm, whereas for the Omegaven ${ }^{\circledR}$ arm, values were recalculated using the relative risks/LOS distributions derived from an earlier meta-analysis of data from 23 randomized controlled European trials in ICU or elective surgery patients receiving PN $(n=1,502)$. In this meta-analysis, omega-3 PUFA-enriched lipid emulsions (various formulations and combinations containing fish oils and/or omega-3 triglycerides) were compared with standard nonenriched lipid emulsions (soybean oil with or without olive oil or medium chain triglycerides). The vast majority of subjects included in this meta-analysis are surgical patients (1,395 of 1,502 patients). ${ }^{17}$

Results revealed that omega-3 PUFA-enriched lipid emulsions give a clear advantage in terms of infective complications and recovery times, as indicated by significant reductions in LOS in the ICU and overall hospitalization duration. Mortality was not significantly affected, possibly reflecting a relatively low underlying mortality risk. Other beneficial effects of omega-3 PUFA-enriched lipid emulsions included improved markers of inflammation, lung gas exchange, liver function, and antioxidant status. The clinical benefits of Omegaven ${ }^{\circledR}$ included in the model were the reductions in ward LOS, ICU LOS, and infection rate in the ICU subgroup ( $\mathrm{n}=762$ ). Clinical inputs for the simulation model, alongside the distribution type from which the individual values for the patient-level simulation were sampled, are summarized in Table 1.
Table I Clinical input parameters and distributions used for the simulation

\begin{tabular}{lll}
\hline Parameter & Mean (SD) & Fixed distribution \\
\hline LOS pre-ICU & $5.3 I(6.72)$ days & Weibull \\
LOS ICU & $10.4(10.1)$ days & Weibull \\
LOS post-ICU & $14.6(14.2)$ days & Weibull \\
Mortality in ICU & $0(0-20$ in SA)\% & Dirichlet \\
Transfer from ICU to ward & $99 \%$ & Dirichlet \\
Discharge from ICU to home & $1 \%$ & Dirichlet \\
Mortality in ward & $7 \%$ & Dirichlet \\
Infection rate & $20 \%$ & Beta \\
Duration of PN & $8.3(10)$ days & Weibull \\
RR infection & $0.7 I(0.17)$ & Gamma \\
$\Delta$ Hospital LOS $^{a}$ & $-5.17(1.62)$ days & Normal \\
$\Delta$ ICU LOS $^{\mathrm{a}}$ & $-1.92(0.69)$ days & Normal \\
\hline
\end{tabular}

Note: ${ }^{a}$ Omegaven ${ }^{\circledR}$ versus standard arm.

Abbreviations: ICU, intensive care unit; SD, standard deviation; SA, sensitivity analysis; PN, parenteral nutrition; RR, relative risk; LOS, length of stay.

\section{Cost inputs}

Unit cost inputs (in renminbi, RMB, 2010 value) for the model were obtained from regression analyses of cost data from the same Chinese hospital dataset. Costs considered were the total hospital cost billed to the patients/relatives, aggregated into the following categories: PN costs, antibiotics costs, drug costs (also including antibiotics), and costs accrued in the ICU (including drugs and PN).

Our aim was to obtain the distributions of the cost of 1 day in ward, 1 day in ICU, 1 day of standard PN, and 1 day of PN with Omegaven ${ }^{\circledR}$ as adjunctive lipid component and of 1 episode of nosocomial infection, in order to reflect the cost structure of the simulation model. ${ }^{22}$ To this aim, the full ICU patient dataset (ie, both arms, differently than in the clinical outcomes analyses) was analyzed and total reported hospital costs were split into three categories: PN costs, antibiotics costs, and stay costs (including all other cost items, detailed by ICU and ward stay).

The cost per day of PN was obtained by calculating individual ratios between $\mathrm{PN}$ cost and $\mathrm{PN}$ duration.

To separate the antibiotic costs for prophylaxis from those for treatment of an infection, we formulated a linear regression model that specified total antibiotics cost as depending on the LOS in ward, the LOS in ICU, and the occurrence of a nosocomial infection. In the simulation model, the cost of infection is limited to the extra antibiotics used for its treatment, disregarding any effect on LOS, which is already captured by the relative LOS estimate in the meta-analysis.

The resulting mean costs for antibiotics used as prophylaxis in ICU and ward, are added to the corresponding stay cost to obtain the full cost of 1 day in ICU and ward, PN excluded. 
Determination of the cost of stay was carried out by specifying total hospital cost (antibiotics and PN excluded) as depending on the LOS in ward and ICU. The cost of the relevant $\mathrm{PN}$ regimen is added to these daily costs.

\section{Sensitivity analysis}

Probabilistic sensitivity analysis (PSA), to assess the effects of uncertainty surrounding the parameter values on the final results, was performed by drawing all parameter values from their probability distributions.

\section{Model validation}

The results predicted by the model were compared with actual data obtained from the patients in the hospital database (ie, those used to feed the baseline clinical parameters and cost inputs of the model). Of particular interest are the clinical results from the Omegaven ${ }^{\circledR}$-treated patients, as these were not used in the modeling, thus permitting an external validation of the model's predictive accuracy.

\section{Results and discussion}

Unit costs obtained from the regression analyses (Table 2) and used for the simulation are summarized in Table 3.

The main simulation results are presented in Table 4. Omega-3 PUFA-enriched lipid emulsions reduce the infection rate (1,348 versus 1,962 infections per 10,000 patients) and the time spent in the ICU and in the hospital overall (24 versus 30 days). Due to shorter LOS (approximately 6 days shorter) and lower infection rates in the Omegaven ${ }^{\circledR}$ arm, the costs related to hospitalization are reduced sufficiently to completely offset the extra treatment costs of 1,793 RMB. Indeed, Omegaven ${ }^{\circledR}$ use results in overall mean cost savings of more than 10,000 RMB.

Omegaven ${ }^{\circledR}$ is expected to dominate standard PN in this patient population, as it is associated with a mean increase

Table 2 Unit costs from regression analyses

\begin{tabular}{|c|c|c|c|}
\hline Parameter & Estimate (RMB) & Standard error & $\boldsymbol{P}$ \\
\hline \multicolumn{4}{|c|}{ Model I: antibiotic costs dependent on infection risk + ICU LOS + } \\
\hline \multicolumn{4}{|c|}{ Ward LOS } \\
\hline Infection & $3,895.83$ & 346.56 & $<2.2 \mathrm{e}-16$ \\
\hline ICU LOS & 291.92 & 12.83 & $<2.2 \mathrm{e}-16$ \\
\hline Ward LOS & 174.49 & 4.81 & $<2.2 \mathrm{e}-16$ \\
\hline \multicolumn{4}{|c|}{ Model 2: stay cost dependent on ICU LOS + Ward LOS } \\
\hline ICU & $1,969.07$ & 89.5 & $<2.2 \mathrm{e}-16$ \\
\hline Ward & I,375.56 & 34.43 & $<2.2 \mathrm{e}-16$ \\
\hline
\end{tabular}

Notes: aultiple $R$-squared: 0.8088 ; adjusted $R$-squared: 0.8082 ; $F$-statistic: I,548 on 3 and I,098 df; bmultiple $R$-squared: 0.7655 ; adjusted $R$-squared: $0.765 \mathrm{I}$; F-statistic: I,794 on 2 and $\mathrm{I}, 099 \mathrm{df}$.

Abbreviations: RMB, Renminbi; ICU, intensive care unit; LOS, length of stay; $d f$, degrees of freedom.
Table 3 Mean (SD) unit costs (RMB) used for the simulation

\begin{tabular}{llll}
\hline Cost/day & Stay & Antibiotics & Total \\
\hline Ward & $\mathrm{I}, 376(90)$ & $174(\mathrm{I3})$ & $\mathrm{I}, 550(90)$ \\
ICU & $\mathrm{I}, 969(34)$ & $292(5)$ & $2,26 \mathrm{I}(35)$ \\
PN: standard arm & & & $655(\mathrm{I})$ \\
PN: Omegaven ${ }^{\circledR}$ arm & & & $\mathrm{I}, 023(\mathrm{I})$ \\
Infection (per episode) & & & $3,896(347)$ \\
\hline
\end{tabular}

Abbreviations: SD, standard deviation; RMB, Renminbi; ICU, intensive care unit; PN, parenteral nutrition.

in effectiveness with a concurrent decrease in mean costs. Results of the 1,000 PSA iterations are represented graphically on a cost-effectiveness plane in Figure 2. Most observations (88\%) are in the south-west quadrant, indicating negative incremental costs and negative incremental infections, ie, showing dominance of the Omegaven ${ }^{\circledR}$ arm.

The results of the comparison are presented in Figures 3 and 4 . The model appears to have good predictive validity in the Chinese ICU setting. All observations are reasonably close to the mean of model predictions and fall well within the $95 \%$ confidence interval (CI) of the values predicted by the PSA. It appears that the estimated LOS differences are a bit conservative, at least when compared to this particular population of patients in the Zhongshan Hospital, whereas the observed cost difference is slightly lower than the predicted mean. In any case, the main findings of the simulation and of the observation coincide, indicating improved patient outcomes at reduced mean costs.

There were no deaths in the studied patient population, which may be surprising in the ICU, where mortality rates are generally high. However, these patients were elective

Table 4 Cost and effectiveness results for ICU patients expressed as mean (SE)

\begin{tabular}{|c|c|c|c|}
\hline & $\begin{array}{l}\text { Omegaven }^{\circledast} \\
\text { arm }\end{array}$ & $\begin{array}{l}\text { Standard } \\
\text { arm }\end{array}$ & Differences \\
\hline \multicolumn{4}{|l|}{ Effectiveness } \\
\hline LOS-total (days) & $24(4.0)$ & $30(0.3)$ & $6.5(4.0)$ \\
\hline Pre-ICU ward & $5.1(0.1)$ & $5.1(0.1)$ & $0(0.0)$ \\
\hline $\mathrm{ICU}$ & 7.7 (1.6) & $10.6(0.2)$ & $-2.9(1.6)$ \\
\hline Post-ICU ward & $10.9(3.6)$ & $14.5(0.2)$ & $-3.6(3.6)$ \\
\hline $\begin{array}{l}\text { Infections/ } \\
\text { I0,000 patients }\end{array}$ & I,348 (453) & I,962 (427) & $-614(362)$ \\
\hline Costs (RMB) & & & \\
\hline Total & $49,219(7,256)$ & $59,836(1,339)$ & $-10,617(7,202)$ \\
\hline $\mathrm{ICU}$ & $\mid 7,960(3,65 \mid)$ & $24,50 \mathrm{I}(\mathrm{I}, \mathrm{II})$ & $-6,54 \mid(3,582)$ \\
\hline Ward (pre-ICU) & $8,185(226)$ & $8,185(226)$ & $0(0)$ \\
\hline Ward & $16,460(5,644)$ & $22,095(552)$ & $-5,635(5,628)$ \\
\hline Infection & $566(185)$ & $80 I(I 78)$ & $-235(142)$ \\
\hline PN & $6,047(526)$ & 4,254 (39) & I,793 (525) \\
\hline
\end{tabular}

Abbreviations: ICU, intensive care unit; SE, standard error; LOS, length of stay, $\mathrm{PN}$, parenteral nutrition. 


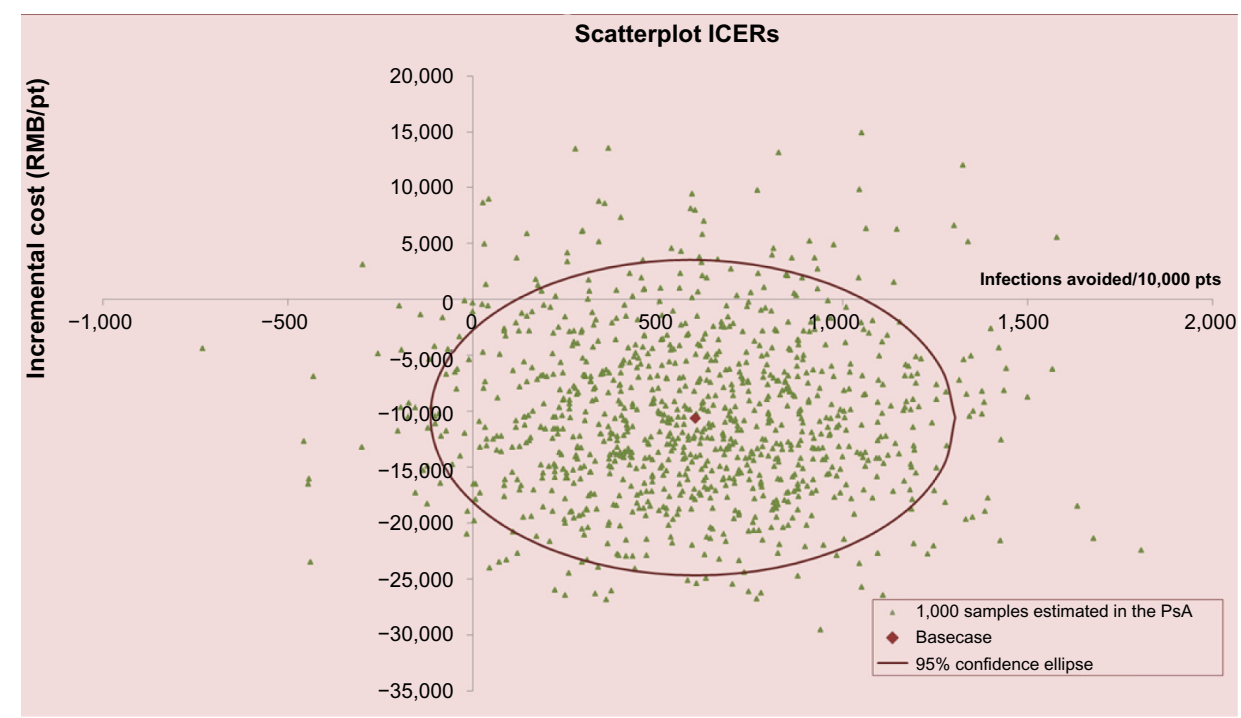

\begin{tabular}{lc}
\hline O-3 versus ST & $\begin{array}{c}\text { Probability } \\
\text { (\%) }\end{array}$ \\
\hline More effective & 96 \\
\hline Lower cost & 92 \\
More effective at lower cost & 88 \\
More effective at increased cost & 8 \\
Lower cost but less effective & 4 \\
\hline Higher cost and less effective & 0 \\
\hline
\end{tabular}

Figure 2 Scatterplot of the incremental cost and avoided infections for the Omegaven ${ }^{\circledR}$ versus the standard arm on a cost-effectiveness plane. Abbreviations: O-3, Omegaven-3; ST, standard; ICER, incremental cost-effectiveness ratio; pt, patient; PSA, probabilistic sensitivity analyses.

surgery patients mainly admitted to the ICU for monitoring and weaning. In this setting, mortality is generally less than $5 \%$, according to both the elective surgery patient group examined by a large meta-analysis, ${ }^{17}$ and the official statistics in Progetto Margherita in Italy, one of the largest ICU outcomes monitoring systems that we are aware of. ${ }^{23}$ On the other, no effect on mortality of the intervention is factored in the model, so low mortality does not introduce bias in the incremental values, although it may affect absolute results.

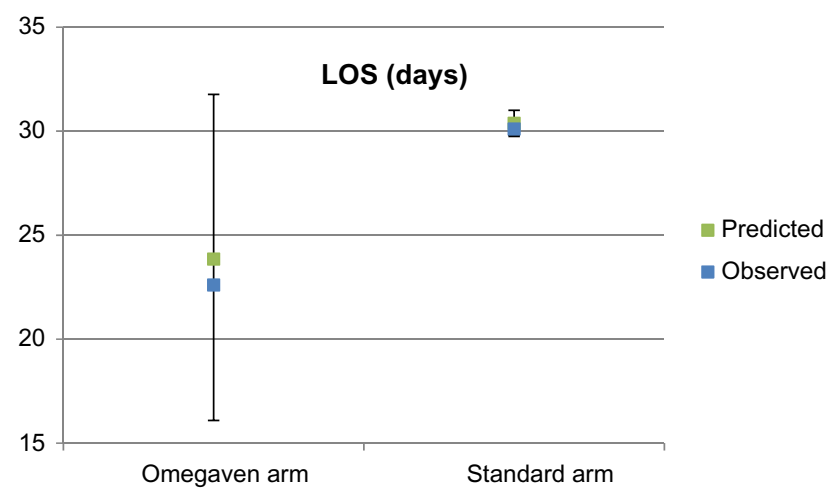

Figure 3 Model validation: observed versus predicted hospital length of stay for the two treatment arms.

Note: Min-max lines represent the $95 \% \mathrm{Cl}$ of predicted values.

Abbreviations: $\mathrm{Cl}$, confidence interval; LOS, length of stay.
A further potential limitation of our analysis is that the relative effectiveness estimates applied to our patient population were derived from international studies conducted on a mixed ICU patient population. Applicability to Chinese standards of care may not be immediately evident. Also, we used Omegaven ${ }^{\circledR}$, which contains $10 \%$ fish oil, in our model, whereas in the meta-analysis, patients received various omega-3 PUFA-enriched lipid emulsions. ${ }^{17}$ Nevertheless, we were able to compare the results predicted by the theoretical

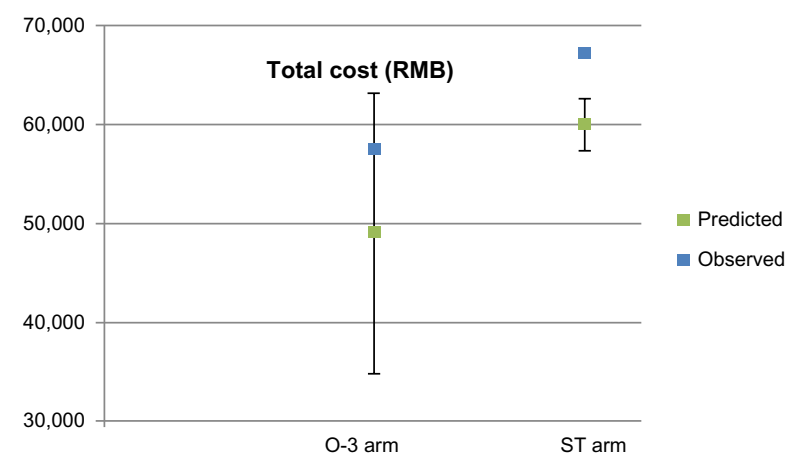

Figure 4 Model validation: observed versus predicted total hospital costs for the two treatment arms.

Note: Min-max lines represent the $95 \% \mathrm{Cl}$ of predicted values.

Abbreviations: $\mathrm{Cl}$, confidence interval; RMB, renminbi; O-3, Omegaven 3; ST, standard. 
model with observed data from the Chinese setting that were not used in the model. This external validation process greatly increases the credibility of these results, which in fact appears partially conservative, as they slightly underestimate the stay reduction observed in our patient population admitted to the ICU.

There is considerable clinical evidence to support the potential of omega-3 PUFA-enriched lipid emulsions to favorably modulate the inflammatory response and, consequently, the outcomes of ICU patients who cannot be fed by the enteral route. ${ }^{17}$ Their acquisition cost is higher than that of standard lipid emulsions, and this could potentially represent a barrier to their widespread adoption. This may be particularly true in the Chinese setting, where the patient (or their family) is asked to pay out of pocket for hospital expenditures. An extra expense for a nutritional product, which may not be perceived as a crucial intervention, may be difficult to accept. However, the results of the present simulation indicate that this initial additional investment for omega-3 PUFA-enriched lipid emulsions should not be of great concern for Chinese ICU patients, since besides hastening recovery, they have the potential to more than offset their extra costs, ultimately resulting in a net saving on the overall expenses for the hospital stay.

\section{Conclusion}

In conclusion, the present results suggest that the appropriate use of omega-3 PUFA-enriched lipid emulsions in PN regimens given to Chinese surgical ICU patients may induce a pattern of faster recovery and subsequent hospitalization cost reduction, as already expected in European settings. ${ }^{22}$

Apart from peculiarities in terms of case mix and clinical practice, it is the underlying positive effect of their use on discharge times that drives the potential to reduce hospitalization costs. This effect can be seen as an overall indicator of the efficacy and safety of the intervention, as reported in the international literature and shown valid when applied to a Chinese hospital setting. ${ }^{20}$

We therefore conclude that the extra costs of omega-3 PUFA-enriched lipid emulsions in the ICU population should not present a barrier to their use and that they should be offered to patients whenever clinically indicated, as their economic convenience goes alongside their clinical utility.

\section{Acknowledgments}

Julia Balfour, Dundee, Scotland, provided editorial assistance for the manuscript. The study was financially supported by Fresenius Kabi.

\section{Disclosure}

LP is employee and co-owner of AdRes, which has received financial support for the analyses and manuscript drafting of this article by Fresenius Kabi. The other authors declare that there is no conflict of interests regarding the publication of this article.

\section{References}

1. Russell C, Elia M. Nutrition Screening Week in the UK and Republic of Ireland in 2011. Hospitals, Care Homes and Mental Health Units. Redditch, UK: BAPEN; 2012. Available from: http://www.bapen.org. uk/professionals/publications-and-resources/bapen-reports. Accessed May 14, 2015.

2. Russell C, Elia M. Nutrition Screening Survey in the UK in 2008. Hospitals, Care Homes and Mental Health Units. Redditch, UK: BAPEN; 2009. Available from: http://www.bapen.org.uk/professionals/ publications-and-resources/bapen-reports. Accessed May 14, 2015.

3. Schindler K, Pernicka E, Laviano A, et al. How nutritional risk is assessed and managed in European hospitals: a survey of 21,007 patients findings from the 2007-2008 cross-sectional nutritionDay survey. Clin Nutr. 2010;29(5):552-559.

4. Meijers JM, Schols JM, van Bokhorst-de van der Schueren MA, Dassen T, Janssen MA, Halfens RJ. Malnutrition prevalence in the Netherlands: results of the annual Dutch national prevalence measurement of care problems. Br J Nutr. 2009;101(3):417-423.

5. Russell C, Elia M. Nutrition Screening Survey and Audit of Adults on Admission to Hospitals, Care Homes and Mental Health Units. Redditch, UK: BAPEN; 2008. Available from: http://www.bapen.org.uk/professionals/publications-and-resources/bapen-reports. Accessed May 14, 2015.

6. Russell C, Elia M. Nutrition Screening Week in the UK and Republic of Ireland in 2010. Hospitals, Care Homes and Mental Health Units. Redditch, UK: BAPEN; 2011. Available from: http://www.bapen.org. uk/professionals/publications-and-resources/bapen-reports. Accessed May 14, 2015.

7. Liang X, Jiang ZM, Nolan MT, et al. Nutritional risk, malnutrition (undernutrition), overweight, obesity and nutrition support among hospitalized patients in Beijing teaching hospitals. Asia Pac J Clin Nutr. 2009;18(1):54-62.

8. Liang X, Jiang ZM, Nolan MT, Efron DT, Kondrup J. Comparative survey on nutritional risk and nutritional support between Beijing and Baltimore teaching hospitals. Nutrition. 2008;24:969-976.

9. Lim SL, Ong KC, Chan YH, Loke WC, Ferguson M, Daniels L. Malnutrition and its impact on cost of hospitalization, length of stay, readmission and 3-year mortality. Clin Nutr. 2012;31(3):345-350.

10. Sorensen J, Kondrup J, Prokopowicz J, et al. EuroOOPS: an international, multicentre study to implement nutritional risk screening and evaluate clinical outcome. Clin Nutr. 2008;27:340-349.

11. Schneider SM, Veyres P, Pivot X, et al. Malnutrition is an independent factor associated with nosocomial infections. Br J Nutr. 2004;92(1): $105-111$.

12. Elia M, Stratton R, Russell C, Green C, Pan F. The Cost of Diseaserelated Malnutrition in the UK and Economic Considerations for the Use of Oral Nutritional Supplements (ONS) in Adults. Redditch, UK: BAPEN; 2005. Available from: http://www.bapen.org.uk/professionals/ publications-and-resources/bapen-reports. Accessed May 14, 2014.

13. Stratton RJ. Malnutrition: another health inequality? Proc Nutr Soc. 2007;66:522-529.

14. Ljungqvist $\mathrm{O}$, de Man F. Under nutrition - a major health problem in Europe. Nutr Hosp. 2009;24(3):368-370.

15. Jie B, Jiang ZM, Nolan MT, et al. Impact of nutritional support on clinical outcome in patients at nutritional risk: a multicenter, prospective cohort study in Baltimore and Beijing teaching hospitals. Nutrition. 2010;26(11):1088-1093. 
16. Martin CM, Doig GS, Heyland DK, Morrison T, Sibbald WJ; for the Southwestern Ontario Critical Care Research Network. Multicentre, cluster-randomized clinical trial of algorithms for critical-care enteral and parenteral therapy (ACCEPT). CMAJ. 2004;170(2): 197-204.

17. Pradelli L, Mayer K, Muscaritoli M, Heller AR. n-3 fatty acid-enriched parenteral nutrition regimens in elective surgical and ICU patients: a meta-analysis. Crit Care. 2012;16(5):R184. (A correction for this article has been published in Crit Care. 2012;17:405).

18. Singer P, Berger MM, Van den Berghe G, et al. ESPEN Guidelines on Parenteral Nutrition: intensive care. Clin Nutr. 2009;28:387-400.

19. Brager M, Ljundqvist O, Soeters P, Fearon K, Weimann A, Bozetti F. ESPEN Guidelines on Parenteral Nutrition: surgery. Clin Nutr. 2009;28: $378-386$.
20. Gao J, Ji CY, Wu GH. [Use of fish oil lipid emulsion in patients undergoing major surgery and those with systemic inflammatory response syndrome: a cost-effectiveness analysis]. Zhonghua Wei Chang Wai Ke Za Zhi. 2012;15(5):452-456. Chinese.

21. Caro JJ. Pharmacoeconomic analyses using discrete event simulation. Pharmacoeconomics. 2005;23:323-332.

22. Pradelli L, Eandi M, Povero M, et al. Cost-effectiveness of omega-3 fatty acid supplements in parenteral nutrition therapy in hospitals: a discrete event simulation model. Clin Nutr. 2014;33(5):785-792.

23. Progetto MARGHERITA - Rapporto 2009. Gruppo Italiano per la Valutazione degli Interventi in Terapia Intensiva (GIVITI). Sestante Edizioni - Bergamo, 2010. [MARGHERITA Project - 2009 report. Italian Group for the Evaluation of Intensive Therapy Interventions (GIVITI)]. Italian.

\section{Publish your work in this journal}

ClinicoEconomics \& Outcomes Research is an international, peerreviewed open-access journal focusing on Health Technology Assessment, Pharmacoeconomics and Outcomes Research in the areas of diagnosis, medical devices, and clinical, surgical and pharmacological intervention. The economic impact of health policy and health systems organization also constitute important areas of coverage. The manuscript management system is completely online and includes a very quick and fair peer-review system, which is all easy to use. Visit http://www.dovepress.com/testimonials.php to read real quotes from published authors.

Submit your manuscript here: http://www.dovepress.com/clinicoeconomics-and-outcomes-research-journal 